\title{
Long lived coherence in self-assembled quantum dots
}

Birkedal, Dan; Leosson, Kristjan; Hvam, Jørn Märcher

Published in:

Physical Review Letters

Link to article, DOI:

10.1103/PhysRevLett.87.227401

Publication date:

2001

Document Version

Publisher's PDF, also known as Version of record

Link back to DTU Orbit

Citation (APA):

Birkedal, D., Leosson, K., \& Hvam, J. M. (2001). Long lived coherence in self-assembled quantum dots. Physical Review Letters, 87(22), 227401. https://doi.org/10.1103/PhysRevLett.87.227401

\section{General rights}

Copyright and moral rights for the publications made accessible in the public portal are retained by the authors and/or other copyright owners and it is a condition of accessing publications that users recognise and abide by the legal requirements associated with these rights.

- Users may download and print one copy of any publication from the public portal for the purpose of private study or research.

- You may not further distribute the material or use it for any profit-making activity or commercial gain

- You may freely distribute the URL identifying the publication in the public portal

If you believe that this document breaches copyright please contact us providing details, and we will remove access to the work immediately and investigate your claim. 


\title{
Long Lived Coherence in Self-Assembled Quantum Dots
}

\author{
D. Birkedal,* K. Leosson, and J. M. Hvam \\ Research Center COM, Technical University of Denmark, DK-2800 Kogens Lyngby, Denmark
}

(Received 2 July 2001; published 7 November 2001)

\begin{abstract}
We report measurements of ultralong coherence in self-assembled quantum dots. Transient four-wave mixing experiments at $5 \mathrm{~K}$ show an average dephasing time of $372 \mathrm{ps}$, corresponding to a homogeneous linewidth of $3.5 \mu \mathrm{eV}$, which is significantly smaller than the linewidth observed in single-dot luminescence. Time-resolved luminescence measurements show a lifetime of the dot ground state of $800 \mathrm{ps,}$ demonstrating the presence of pure dephasing at finite temperature. The homogeneous width is lifetime limited only at temperatures approaching $0 \mathrm{~K}$.
\end{abstract}

DOI: 10.1103/PhysRevLett.87.227401

PACS numbers: 78.67.Hc, 78.47.+p, 78.66.Fd

Semiconductor quantum dot systems have recently been proposed as the basic building blocks for solid-state-based quantum logic devices due to a well-established processing technology and potential ease of integration with existing devices [1-4]. One of the main obstacles is, however, the relatively short dephasing time $\left(T_{2}\right)$, inherent to the solid state, setting an upper limit to the number of possible quantum operations. Recent single-dot photoluminescence (PL) experiments on III-V self-assembled quantum dots (SAQD) [5-10] and localized excitons in narrow quantum wells $[11,12]$ have shown narrow emission lines indicating an upper limit on $T_{2}$ of $50 \mathrm{ps}$ which is still too short for practical application. The conversion of a PL linewidth to a dephasing time assumes that the single-dot emission is homogeneously broadened. This assumption seems reasonable and has been confirmed in the case of localized excitons in narrow quantum wells, where $T_{2}$ has been measured directly using transient degenerate four-wave mixing (TFWM) [13]. However, in the case of SAQD much longer dephasing times should be expected due to the stronger confinement of the excitons $[14,15]$. TFWM experiments have proven very difficult to perform on SAQD due to the very low signal strength. So far the only experiments have been performed on dot layers processed into waveguides and only at room temperature, where very short $T_{2}$ 's were measured [16].

In this Letter, we present low temperature TFWM experiments showing ultralong $T_{2}$ of $\approx 370$ ps corresponding to a homogeneous width $\Gamma_{h}$ of $3.5 \mu \mathrm{eV}$. This linewidth is considerably narrower than observed in single-dot PL experiments showing that inhomogeneous effects dominate the PL emission line. We furthermore demonstrate that $\Gamma_{h}$ is limited by population decay at temperatures near $0 \mathrm{~K}$. At these temperatures, $T_{2}$ in excess of $1 \mathrm{~ns}$ are attainable, which should enable practical demonstration of quantum logic operations in the solid state.

The samples in the present study consist of $\mathrm{In}_{0.5} \mathrm{Al}_{0.04} \mathrm{Ga}_{0.46} \mathrm{As}$ SAQDs embedded in $\mathrm{Ga}_{0.92} \mathrm{Al}_{0.08} \mathrm{As}$, grown by molecular beam epitaxy. The small amount of $\mathrm{Al}$ is added to tune the transition energy for experimental convenience. A sample with ten layers of SAQD was grown for TFWM and time-resolved PL (TR-PL). Antireflection coating was applied to the sample backside to prevent multiple reflections of the exciting beams. A second sample with a single layer of dots was grown for single-dot PL spectroscopy. A thin gold film with submicron sized apertures, defined using electron beam lithography, was deposited on the surface of the second sample.

For TFWM and TR-PL the sample was cooled in a liquid helium cryostat and excited with 120 fs pulses from a Ti:sapphire laser. In the TFWM experiments we used the $2 \vec{k}_{2}-\vec{k}_{1}$ transmission geometry and detected the signal using a silicon photodiode with a low-noise preamplifier and lock-in techniques. In the TR-PL experiments the sample was excited in the barriers and the PL was collected, dispersed, and synchronously detected using a streak camera with 2.5 ps time resolution.

Single-dot PL experiments were carried out down to $10 \mathrm{~K}$ in a closed-cycle He cryostat. The PL was excited and collected through a microscope objective located inside the cryostat. A He-Ne laser was used for excitation. The PL was dispersed in a 2-m Littrow-type spectrometer with a resolution of $0.1 \AA(\approx 20 \mu \mathrm{eV})$.

We first present our TFWM results. The data shown in the following are recorded at an excitation density of $190 \mathrm{~W} / \mathrm{cm}^{2}$ which is more than 1 order of magnitude larger than we usually use for quantum well samples. However, the absorbance $(\alpha d)$ of quantum dots is rather low due to the low areal coverage. Using the value $\alpha d=$ $2 \times 10^{-3}$ obtained from a six layer sample of similar areal density $\left(2-3 \times 10^{10} \mathrm{~cm}^{-2}\right)$ [17] we find that no more than $15 \%$ of the dots are excited during the experiments. In addition, we find that decay times of the TFWM signal are unaffected by increasing or decreasing the excitation density by a factor of 2 . The TFWM intensity varies with excitation intensity in near perfect agreement with an underlying $\chi^{(3)}$ process.

The TFWM signal recorded at three different spectral positions of the laser $(A, B$, and $C)$ and at a temperature of $5 \mathrm{~K}$ is shown in Fig. 1. The inset shows the 5-K PL spectrum and the spectral positions of the laser for the three TFWM traces. A dominating signal at pulse overlap $(\tau=$ 0 ) is common for the three TFWM traces. This signal is due to a nonresonant two-photon transition to the GaAs 


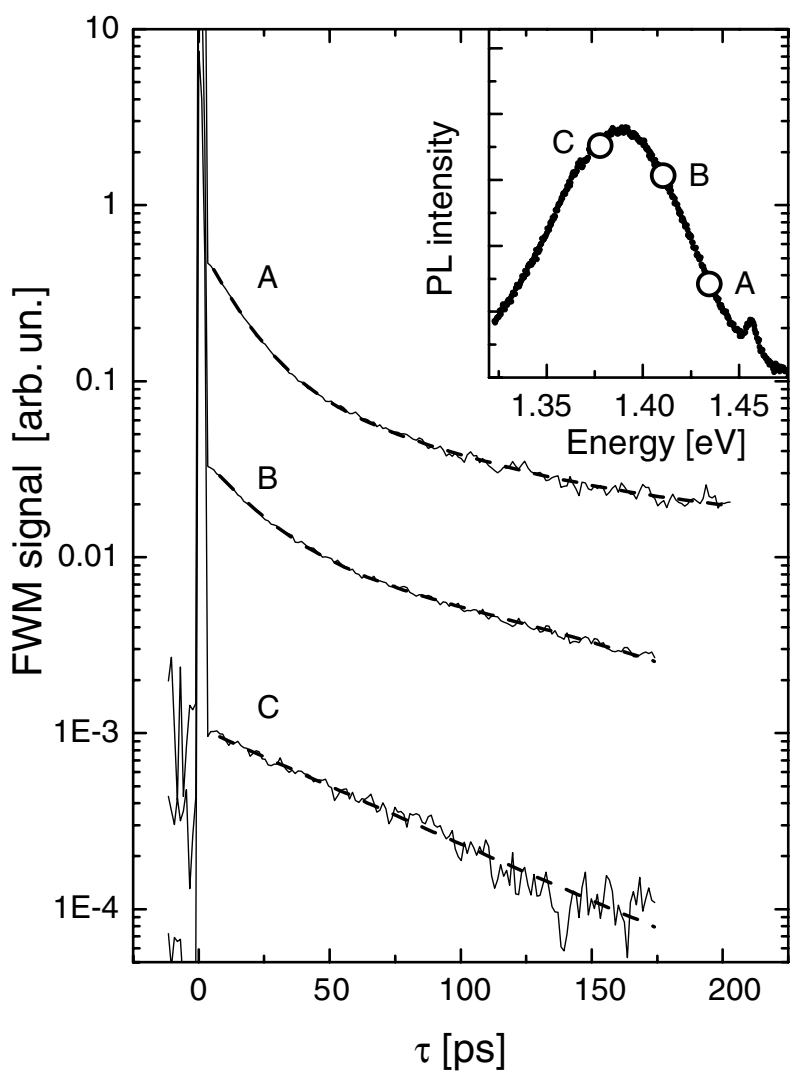

FIG. 1. Transient wave mixing. The data are taken for three different detunings of the laser $(A, B$, and $C)$ as indicated in the inset, which also shows the sample luminescence spectrum. The small PL peak at around $1.45 \mathrm{eV}$ is impurity-related emission from the sample substrate.

substrate and is unrelated to the quantum dots [17]. For longer delays, a much weaker (3-4 orders of magnitude) signal is observed. This weaker signal is seen to depend on the detuning of the laser with respect to the quantum dot PL peak. For positive detuning $(A)$ the TFWM signal is dominated by a fast component for short delays, and a much slower component for longer delays. The influence of the fast component is seen to diminish for more resonant detuning $(B)$ and finally disappear for negative detuning $(C)$. However, the slow component is observed to persist across the entire quantum dot distribution.

To analyze the TFWM data in further detail we have fitted a biexponential decay $\left[I(\tau)=A_{1} \exp \left(-\tau / \tau_{1}\right)+\right.$ $\left.A_{2} \exp \left(-\tau / \tau_{2}\right)\right]$ to the data as indicated by the dashed lines in Fig. 1. The good agreement of the fit with the data demonstrates that two distinct processes contribute to the TFWM signal. To obtain more quantitative information of the origin of the two processes we have recorded the TFWM signal for a number of different spectral positions of the laser. Each of these TFWM traces is fitted with a biexponential decay, and two decay times and two amplitudes are extracted.

The results from the biexponential fits are shown in Figs. 2(a) and 2(b). The two decay-associated amplitudes
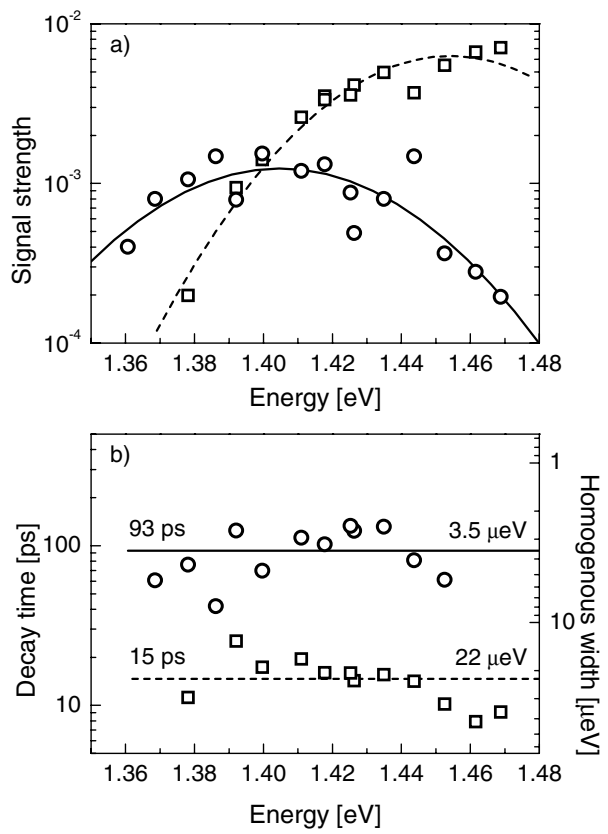

FIG. 2. Decay-associated amplitudes (a) and decay times (b). The amplitude and decay time for the slow component are shown with circles and the amplitude and decay time for the fast component are shown with squares. The lines are (a) Gaussian fits to the data points and are guides to the eye and (b) averages of the data points.

are shown in Fig. 2(a). The amplitude of the slow component has a maximum at $1.405 \mathrm{eV}$, which nearly coincides with the peak of the low temperature PL spectrum (Fig. 1 inset). In contrast, the amplitude of the fast component displays no clear maximum and merges into the onset of the wetting-layer transition. Since the lowtemperature PL line shape is in near agreement with the density of dot ground states (we assume that relaxation from excited states is fast and thermal redistribution of excitons between ground states is absent), we conclude that the slow component of the TFWM signal is due to excitation of dot ground states and the fast component is due to excitation of a mixture of wetting layer and excited states of the dots. To further support this we note that the width of the PL spectrum is nearly equal to the width of the distribution of the decay-associated amplitudes of the slow component in the TFWM signal.

We now turn to the decay times of the two contributions to the TFWM, which are plotted in Fig. 2(b). The decay times of the fast and slow components are clearly separated in the plot. Because of the unavoidable scatter in the data there seems to be no basis for observing a systematic spectral dependence of any of the decay times. Instead we calculate the average decay times, which are indicated with the horizontal lines.

The average of the measured long decay times is 93 ps. Since the TFWM signal is a time-integrated photon echo this decay time corresponds to $T_{2}=372 \mathrm{ps}$. This value is more than an order of magnitude larger than previously 
reported for SAQD [5-10] and excitons localized to potential fluctuations of narrow quantum wells $[11,12]$. The average $T_{2}$ can be converted to a $\Gamma_{h}$ of $3.5 \mu \mathrm{eV}$.

The short decay time has an average of 15 ps or a $T_{2}$ of $60 \mathrm{ps}$, which corresponds to a $\Gamma_{h}$ of $22 \mu \mathrm{eV}$. We speculate that the faster dephasing of the excited states is due to relaxation to the ground states. This channel of relaxation is not available to the ground states, hence the difference of the two times. Assuming that relaxation processes dominate the homogeneous broadening of the excited states this corresponds to an excited state lifetime $T_{1}$ of $30 \mathrm{ps,} \mathrm{which}$ is agreement with our assumption of fast relaxation of the excited states. We note that Toda et al. [10] have reported an even shorter $T_{2}$ of $15 \mathrm{ps}$ for the excited states of SAQD. This value was obtained in a near field experiment and we speculate that the faster dephasing is due to the proximity of the dots to the surface in the particular sample.

We can now conclude that $\Gamma_{h}$ of the ground states of our SAQD is approximately 1 order of magnitude smaller than reported so far in single-dot PL spectroscopy. This indicates that the linewidth obtained in single-dot spectroscopy is not necessarily $\Gamma_{h}$ as often argued. To make a direct comparison on our SAQDs we have performed single-dot spectroscopy on the apertured single-dot-layer sample.

The 10-K PL spectrum from a 500-nm aperture is shown in the left inset of Fig. 3. The spectrum consists of a large number of well-resolved single lines. A selection of the spectrum indicated by the rectangular box is recorded at high spectral resolution and shown in Fig. 3. The lines are well approximated with Lorentzians as shown with the solid line. The width (FWHM) is extracted for 54 lines and shown in the linewidth histogram in the right inset of Fig. 3. Other apertures show similar linewidth statistics. The most common measured linewidth is $\approx 65 \mu \mathrm{eV}$. When corrected for the spectral resolution $(20 \mu \mathrm{eV})$ we obtain a typical dot linewidth of $\approx 45 \mu \mathrm{eV}$, which is in

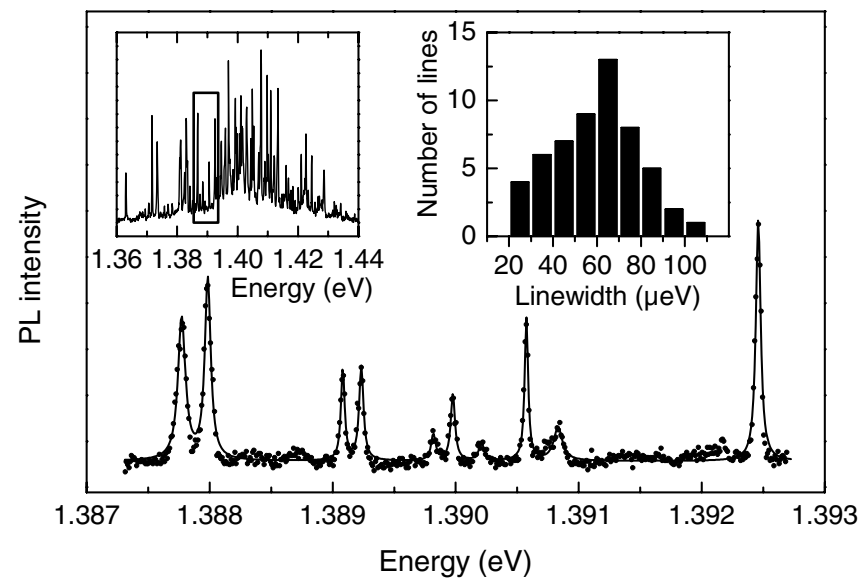

FIG. 3. High-resolution photoluminescence spectrum obtained through a $500-\mathrm{nm}$ aperture at $5 \mathrm{~K}$. The solid line is a Lorentzian fit to the data. The left inset shows all the lines from the 500-nm aperture. The right inset shows the linewidth statistics. good agreement with published data on linewidths from self-organized quantum dots [5-10].

We have performed temperature dependent single-dot PL measurements to be able to correct for the temperature difference between single-dot and TFWM experiments. We find that the width of the single-dot lines are well described by $\Gamma(T)=\Gamma(0)+a T$ up to a temperature of $\approx 40 \mathrm{~K}$. We find that $a \approx 0.5 \mu \mathrm{eV} / \mathrm{K}$ and $\Gamma(0)$ is described by a distribution peaking at $\approx 40 \mu \mathrm{eV}$. We have also investigated the temperature dependence of the TFWM signal and have been able to acquire data up to a temperature of $40 \mathrm{~K}$. It is found that $\Gamma_{h}(T)=\Gamma_{h}(0)+$ $\alpha T$ with $\alpha \approx 0.5 \mu \mathrm{eV} / \mathrm{K}$ for both the dot ground states and excited states. This means that $\Gamma_{h}(0) \approx 1 \mu \mathrm{eV}$ for the dot ground state, corresponding to a $T_{2}$ of more than a ns. For temperatures higher than $40 \mathrm{~K}$ the intensity of the TFWM becomes too weak to extract reliable values of the decay times.

By comparing the extrapolated $0-\mathrm{K}$ linewidths obtained in single-line PL experiments with $\Gamma_{h}(0)$ from the TFWM experiments we reach a general conclusion: Single-dot PL linewidths can in general not be taken as $\Gamma_{h}$. They are merely an upper bound on $\Gamma_{h}$. The difference is easy to understand if we consider that single-dot PL experiments often are carried out using substantial integration times and thus many emission events. Perturbations, such as capture of excitons or charged particles into neighboring dots, can shift the emission energy from event to event thus introducing significant broadening [18-21].

Localized excitons in narrow quantum wells have also been investigated using single-line spectroscopy and have shown linewidths in the range of $22-50 \mu \mathrm{eV}[11,12]$, which have been interpreted as $\Gamma_{h}$. However, in the case of localized excitons in narrow quantum wells the conclusion is supported by TFWM investigations where $T_{2} \approx 50 \mathrm{ps}$ were found, corresponding to $\Gamma_{h} \approx 27 \mu \mathrm{eV}$ [13]. The authors furthermore found that $T_{2}$ at low temperature was dominated by the exciton lifetime $T_{1}$, which is shorter in quantum well structures than in dot systems with stronger confinement [13,15].

Since $\Gamma_{h}$ of localized excitons in narrow quantum wells is dominated by $T_{1}$ at the lowest temperatures it is natural to ask whether the same situation applies for SAQD. To answer this question we have performed TR-PL spectroscopy on the ten-layer sample. This allows us to evaluate the PL decay time for individual spectral components across the entire luminescence band. We show in Fig. 4 the timeintegrated PL spectrum together with the spectrally resolved decay times. The data show a spectral dependence of the PL decay times from $650 \mathrm{ps}$ at the high-energy side to $850 \mathrm{ps}$ at the low-energy side of the PL spectrum.

The time-integrated PL spectrum coincides with the PL obtained under continuous-wave excitation. The PL spectrum and PL decay times are nearly independent of excitation density over 2 orders of magnitude. We therefore conclude that the decay times observed are the lifetimes 


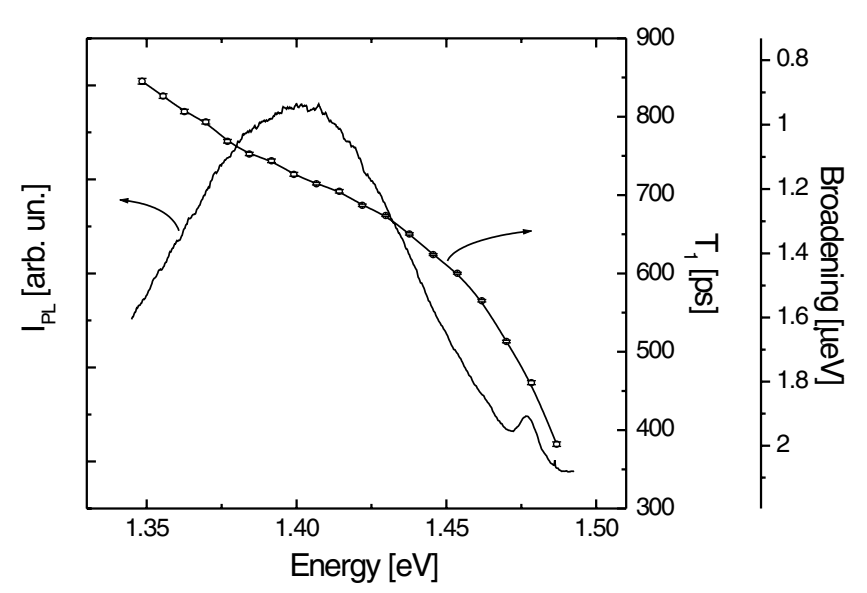

FIG. 4. Time-resolved photoluminescence. The left axis shows the time-integrated photoluminescence and the first right axis shows measured population decay times $T_{1}$ at different spectral positions. The second right axis shows the same data on an energy scale.

$T_{1}$ of the dot ground states. This allows us to calculate the contribution to $\Gamma_{h}$ from population decay, which is shown on the rightmost vertical axis of Fig. 4. We find that population decay gives rise to a linewidth of $\approx 1 \mu \mathrm{eV}$, which is in agreement with $\Gamma_{h}(0)$ obtained from TFWM experiments. This shows that $\Gamma_{h}$ of SAQD is limited by population decay at zero temperature. However, the dominance of pure dephasing is already established at a temperature of 3-4 K. The linear dependence of $\Gamma_{h}$ with temperature is consistent with the theory of Takagahara [15] indicating that acoustic phonons could be responsible for the pure dephasing.

In summary, we have reported TFWM experiments on self-assembled quantum dots. We have shown ultralong coherence of the dot ground state, which is more than 1 order of magnitude longer than found for localized exci- tons in narrow quantum wells. We corroborate that dephasing times in excess of $1 \mathrm{~ns}$ are attainable in self-assembled quantum dots, which should enable the demonstration of quantum logic operations in the solid state. Finally, we find that the PL linewidth from single self-assembled quantum dots is significantly wider than the homogenous linewidth, showing that inhomogeneous processes dominate the PL linewidth.

The authors thank C. B. Sørensen III-V Nanolab and J.R. Jensen for sample growth and K. Yvind for antireflection coating.

*Electronic address: Birkedal@com.dtu.dk

[1] A. Ekert and R. Jozsa, Rev. Mod. Phys. 68, 733 (1996).

[2] D. Loss and D. P. DiVincenzo, Phys. Rev. A 57, 120 (1998).

[3] A. Imamoglu et al., Phys. Rev. Lett. 83, 4204 (1999).

[4] G. Chen et al., Science 289, 1906 (2000).

[5] H. Kamada et al., Jpn. J. Appl. Phys. 36, 4194 (1997).

[6] K. Ota, N. Usami, and Y. Shiraki, Physica (Amsterdam) 2E, 573 (1998).

[7] K. Asaoka et al., Jpn. J. Appl. Phys. 38, 546 (1999).

[8] Y. Toda et al., Appl. Phys. Lett. 73, 517 (1998).

[9] Y. Toda et al., Phys. Rev. Lett. 82, 4114 (1999).

[10] Y. Toda et al., Appl. Phys. Lett. 76, 3887 (2000).

[11] D. Gammon et al., Phys. Rev. Lett. 76, 3005 (1996).

[12] D. Gammon et al., Science 273, 87 (1996).

[13] X. Fan et al., Solid State Commun. 108, 857 (1998).

[14] T. Takagahara, Phys. Rev. Lett. 71, 3577 (1993).

[15] T. Takagahara, Phys. Rev. B 60, 2638 (1999).

[16] P. Borri et al., Phys. Rev. B 60, 7784 (1999).

[17] D. Birkedal et al., Appl. Phys. Lett. 77, 2201 (2000).

[18] R. G. Neuhauser et al., Phys. Rev. Lett. 85, 3301 (2000).

[19] H.D. Robinson and B. B. Goldberg, Phys. Rev. B 61, R5086 (2000).

[20] P. G. Blome et al., Phys. Rev. B 61, 8382 (2000).

[21] J. Seufert et al., Appl. Phys. Lett. 76, 1872 (2000). 